\section{O idoso institucionalizado: avaliação da capacidade funcional e aptidão física}

\author{
Institutionalized elderly: functional capacity \\ and physical fitness
}

\author{
Lúcia Hisako Takase Gonçalves 1 \\ Aline Huber da Silva ${ }^{2}$ \\ Giovana Zarpellon Mazo ${ }^{3}$ \\ Tânia R. Bertoldo Benedetti 4 \\ Silvia Maria Azevedo dos Santos 1 \\ Sueli Marques 5 \\ Rosalina A. Partezani Rodrigues 5 \\ Marilene Rodrigues Portella 6 \\ Helenice de Moura Scortegagna 6 \\ Silvana Sidney C. Santos 7 \\ Marlene Teda Pelzer 7 \\ Andrea dos Santos Souza 8 \\ Edmeia Campos Meira ${ }^{8}$ \\ Edite Lago da Silva Sena 8 \\ Marion Creutzberg 9 \\ Tais de Lima Rezende 9
}

\section{Introdução}

Federal de Santa Catarina

Florianópolis, Brasil.

2 Programa Pós-graduação

em Ciências do Movimento

Humano, Universidade do

Estado de Santa Catarina,

Florianópolis, Brasil.

${ }^{3}$ Centro de Ciências da Saúde

e do Esporte, Universidade

do Estado de Santa Catarina,

Florianópolis, Brasil.

${ }_{4}^{4}$ Centro de Desportos,

Universidade Federal

de Santa Catarina

Florianópolis, Brasil.

5 Escola de Enfermagem de

Ribeirão Preto, Universidade

de São Paulo, Ribeirão Preto,

Brasil.

${ }^{6}$ Faculdade de Enfermagem,

Universidade de Passo Fundo,

Passo Fundo, Brasil.

7 Departamento de

Enfermagem, Fundação

Universidade Federal do Rio

Grande, Rio Grande, Brasil.

8 Universidade Estadual do

Sudoeste da Bahia, Jequié,

Brasil.

9 Pontifícia Universidade

Católica do Rio Grande do

Sul, Porto Alegre, Brasil.

Correspondência

L. H. T. Gonçalves

Departamento de

Enfermagem, Universidade

Federal de Santa Catarina.

Campus Universitário,

Florianópolis, SC

88040-900, Brasil.

lucia.takase@pq.cnpq.br
This study analyzed the relationship between physical fitness and functional capacity in 78 residents of long-stay institutions for low-income elderly located in five regions of Brazil. The majority of the sample consisted of women, and mean age was 77.4 years $(S D=7.9)$. Physical fitness was assessed with the AAHPERD test, adjusted for institutionalized elderly. The Katz scale was used for functional capacity. The five components of physical fitness rated fair for flexibility, coordination, agility, and aerobic endurance and good for strength. The mean general physical fitness (GPF) index was fair. According to the findings, the greater the degree of dependency in institutionalized elderly, the lesser their strength and GPF level; meanwhile, better coordination and agility are associated with greater independence for performing activities of daily living. The results can contribute to appropriate physical exercise programs for maintenance and/or recovery offunctionality.

\section{Physical Fitness; Homes for the Aged; Aged}

Abstract
A institucionalização não se constitui ainda uma prática comum em nossa sociedade. Segundo o Censo Demográfico de 2000, havia 103 mil idosos residentes em domicílios coletivos incluindo os asilos, representando em torno de $0,8 \%$ da população idosa 1. Contudo, fatores demográficos, sociais e de saúde constituem-se em causas que tendem a levar idosos a residir em instituições de longa permanência para idosos, denominação atual para o tradicional asilo 2 . Acredita-se que, entre outros motivos, a participação feminina no mercado de trabalho retira do domicílio a figura tradicionalmente convocada para o cuidado dos pais ou sogros. As mudanças na nupcialidade e novos arranjos familiares também reduzem a perspectiva de envelhecer em um ambiente familiar ${ }^{3}$.

O processo de envelhecimento, por si só, pode acarretar o declínio da aptidão física e da capacidade funcional 4,5 , que se agrava com o sedentarismo, tornando os idosos dependentes de cuidados de outrem. Observa-se que tal situação prevalece mais entre os idosos institucionalizados, tornando-os detentores de várias conseqüências decorrentes da inatividade 5,6.

Por isso, a avaliação da capacidade funcional e da aptidão física dos idosos para orientar intervenções específicas e seu acompanhamento é fundamental no combate das dependências 
preveníveis e na promoção de uma vida mais ativa possível. A detecção do grau de dependência do idoso nos diferentes componentes da aptidão física possibilita selecionar intervenções adequadas, aplicadas tanto individualmente quanto em grupo, com vistas à melhoria da capacidade funcional principalmente no desempenho das atividades da vida diária.

Tais avaliações e intervenções sistematizadas em instituições de longa permanência para idosos não representam ainda um procedimento usual 7 , como também são poucos os estudos que avaliam as condições físicas e funcionais dos idosos institucionalizados 8,9. Com relação à aptidão física, as pesquisas são ainda mais escassas. Portanto, avaliar a capacidade funcional e a aptidão física dos idosos institucionalizados e verificar sua possível relação são requisitos essenciais para implantar um adequado programa de exercícios e atividades físicas, com ênfase nos diferentes componentes da aptidão física dos residentes em instituições de longa permanência para idosos.

Assim, o objetivo deste estudo foi explorar a relação entre a aptidão física e a capacidade funcional de residentes em instituições de longa permanência para idosos de baixa renda.

\section{Metodologia}

O estudo fez parte da pesquisa multicêntrica, interinstitucional Instituições de Longa Permanência para Idosos de Baixa Renda: Proposta de Modelo Básico de Assistência Multidimensional, envolvendo seis universidades que se propuseram a participar do projeto, localizadas em diferentes municípios, distribuídos em três regiões geográficas do país: (1) Região Sul - Universidade Federal de Santa Catarina (UFSC) em Florianópolis, Santa Catarina; Pontifícia Universidade Católica do Rio Grande do Sul (PUCRS) em Porto Alegre, Rio Grande do Sul; Universidade Federal do Rio Grande (FURG) em Rio Grande, Rio Grande do Sul; Universidade de Passo Fundo (UPF) em Passo Fundo, Rio Grande do Sul; (2) Região Sudeste - Escola de Enfermagem de Ribeirão Preto, Universidade de São Paulo (EERP-USP) em Ribeirão Preto, São Paulo; (3) Região Nordeste - Universidade Estadual do Sudoeste da Bahia (UESB) em Jequié, Bahia.

O projeto comprometia-se a cumprir todas as exigências éticas de respeito humano previstas na Resolução $n^{\circ}$. 196/96 do Conselho Nacional de Saúde, foi submetido e aprovado pelo Comitê de Ética em pesquisa com seres humanos da UFSC, e protocolado sob o $\mathrm{n}^{\mathrm{o}}$. 013/07 em 26 de março de 2007. Os idosos selecionados aceitaram parti- cipar do estudo e assinaram o Termo de Consentimento Livre e Esclarecido.

A implementação da proposta transcorreu por um período de dois anos e meio (2007-2009), mas os dados específicos da presente análise foram colhidos no primeiro semestre de 2008. Em cada município selecionou-se uma instituição de longa permanência para idosos a critério da equipe de pesquisa local, que atendesse a condição de ser uma instituição filantrópica para idosos de baixa renda e cujo dirigente aceitasse participar do estudo. Após a seleção das seis instituições de longa permanência para idosos, identificou-se que a população alvo contava com 377 residentes, distribuídos em: 45 idosos na instituição de Florianópolis, 150 na de Porto Alegre, 42 na de Passo Fundo, 50 na de Rio Grande, 40 na de Ribeirão Preto e 50 na de Jequié. Contudo, para definir a amostra foram adotados os seguintes critérios de inclusão: os idosos deveriam ter realizado os cinco testes da bateria AAHPERD (American Alliance for Health, Physical Education, Recreation and Dance) e a avaliação da capacidade funcional; não ser cadeirante; não ser acamado e/ou classificado no grau III de dependência (segundo a Resolução $n^{\circ}$. 283/05 10, dependência que requer assistência em todas as atividades de autocuidado); e não estar em grau avançado de demência. Foram identificados 92 idosos dentro dos critérios adotados, mas ao aplicarmos a AAHPERD, 14 (13\%) deles não conseguiram completar os cinco testes da bateria. Assim, a amostra constituiu-se de 78 idosos, com média de idade de 77,37 (DP = 7,87), nas seis instituições de longa permanência para idosos estudadas: 10 idosos de Florianópolis, 33 de Porto Alegre, 4 de Passo Fundo, 9 de Rio Grande, 7 de Ribeirão Preto e 15 de Jequié.

Os instrumentos de coleta de dados foram: a escala de Katz 11,12, que avalia a capacidade funcional para o desempenho das atividades básicas da vida diária, compreendendo o banharse, vestir-se, ir ao banheiro, fazer transferência da cama à cadeira e vice-versa, continência dos esfincteres, e alimentar-se; e a bateria de testes físicos desenvolvida pela AAHPERD 13 que avalia a aptidão física dos idosos, que foi adaptada para idoso institucionalizado 8 .

Essa bateria é composta de cinco testes físicos que avaliam os componentes: agilidade/equilíbrio dinâmico; coordenação; flexibilidade de membros inferiores; força dos membros superiores; resistência aeróbia geral e habilidade de andar. Quanto à referida adaptação da bateria, deuse no teste de flexibilidade e de resistência aeróbia. No teste de flexibilidade, em lugar de sentarse no chão para realizá-lo, uma tarefa árdua para idosos, a adaptação se constituiu de uma tábua apoiada em duas cadeiras, onde o idoso sentado 
em outra cadeira estendeu as pernas sobre a tábua para realizá-lo. O teste de resistência aeróbia, que consiste em caminhar meia-milha $(804,67 \mathrm{~m})$, foi substituído pelo teste de caminhada de $6 \mathrm{mi}$ nutos (TC6M), considerando a impossibilidade do idoso de andar meia-milha e o espaço físico restrito das instituições de longa permanência para idosos para a sua realização 14 .

A aplicação dos testes de avaliação da capacidade funcional e de aptidão física junto aos idosos residentes foi realizada pelas seis equipes locais de pesquisa, correspondentes às instituições de longa permanência para idosos estudadas, que foram treinadas previamente pela coordenação geral do estudo composta de pesquisadoras da área de educação física e fisioterapia. Um CD de aplicação da AAHPERD adaptada para idosos institucionalizados foi produzido e incluído no programa de treinamento das equipes.

A aptidão física foi avaliada pelos valores percentis dos cinco testes físicos e pelo Índice de Aptidão Física Geral (IAFG) (Tabela 1), que é o somatório dos valores percentis dos cinco testes. Esses valores foram criados e classificados - fraco, regular e bom - com base nos resultados da amostra deste estudo, não podendo ser generalizados para todos os idosos que residem em instituições.

A capacidade funcional foi avaliada pela escala de independência nas atividades da vida diária (escala de Katz). Essa classificação foi indicada pelo Ministério da Saúde 12, pois identifica em qual atividade o indivíduo apresenta a dependência. Ela varia de A a G e OUTRO, em que: A - independente para todas as atividades; $\mathrm{B}$-independente para todas as atividades menos uma; $\mathrm{C}$ - independente para todas as atividades menos banho e mais uma adicional; D - inde- pendente para todas as atividades menos banho, vestir-se e mais uma adicional; E - independente para todas as atividades menos banho, vestir-se, ir ao banheiro e mais uma adicional; $\mathrm{F}$ - independente para todas as atividades menos banho, vestir-se, ir ao banheiro, transferência e mais uma adicional; $\mathrm{G}$ - dependente para todas as atividades; OUTRO - dependente em pelo menos duas funções, mas que não se classificasse em C, D, E e F. Nenhum idoso foi classificado com nível de dependência G e OUTRO.

Por meio do teste Kolmogorov-Smirnov verificou-se que os dados não são normais ( $p=0,029$ ). Diante disso, para correlacionar os diferentes componentes da aptidão física, o IAFG e a capacidade funcional dos idosos institucionalizados optou-se pelo teste de correlação de Spearman (rho), com nível de significância de 5\%.

\section{Resultados}

Na Tabela 2 estão apresentadas as características sociodemográficas, a prática de atividade física, a capacidade funcional, os componentes da aptidão física e o IAFG dos idosos institucionalizados.

Observa-se que a maioria dos idosos institucionalizados é do sexo feminino, tem de 1 a 4 anos de estudos, não pratica atividade física e é independente para as atividades da vida diária Quanto à aptidão física, em seus cinco componentes, em média é regular na flexibilidade, coordenação, agilidade e resistência aeróbia, e boa na dimensão força. Já o IAFG em média é regular.

Verifica-se na Tabela 3 que entre a capacidade funcional e os componentes da aptidão física e o IAFG houve correlação moderada negativa para a

Tabela 1

Valores percentis e classificação da aptidão física e do Índice de Aptidão Física Geral (IAFG) dos idosos residentes em instituições de longa permanência, 2009.

\begin{tabular}{lccc}
\hline Variáveis & Fraca & $\begin{array}{c}\text { Classificação } \\
\text { Regular }\end{array}$ & Boa \\
& & & $\geq 47$ \\
Componentes da aptidão física & & $34-45$ & $\leq 34,29$ \\
Flexibilidade (cm) & $\leq 33$ & $58-34,89$ & $\leq 62$ \\
Coordenação (s) & $\geq 61$ & $85,9-63$ & $\geq 8$ \\
Agilidade (s) & $\geq 86,08$ & $5-7$ & $\geq 297,6$ \\
Força (repetições) & $\leq 4$ & $197,5-290$ & $333-500$ \\
Resistência aeróbia - TC6M (m) & $\leq 193$ & $167-332$ & $0-166$ \\
IAFG & & & \\
\hline
\end{tabular}

$\mathrm{cm}$ : centímetros; m: metros; s: segundos; TC6M: teste de caminhada de 6 minutos. 
Características sociodemográficas, prática de atividade física, capacidade funcional, componentes da aptidão física e Índice de Aptidão Física Geral (IAFG) dos idosos residentes em instituições de longa permanência, 2009.

\begin{tabular}{|c|c|c|}
\hline Variáveis & $\mathbf{n}$ & $\%$ \\
\hline \multicolumn{3}{|l|}{ Características sociodemográficas } \\
\hline \multicolumn{3}{|l|}{ Sexo } \\
\hline Masculino & 34 & 43,6 \\
\hline Feminino & 44 & 56,4 \\
\hline \multicolumn{3}{|l|}{ Escolaridade (anos de estudos) } \\
\hline Analfabeto & 29 & 37,2 \\
\hline 1 a 4 & 34 & 43,6 \\
\hline 2 a 5 & 12 & 15,4 \\
\hline 9 ou mais & 3 & 3,8 \\
\hline \multicolumn{3}{|l|}{ Prática de atividade física } \\
\hline Sim & 1 & 1,3 \\
\hline Não & 77 & 98,7 \\
\hline \multicolumn{3}{|l|}{ Capacidade funcional * } \\
\hline A & 60 & 76,9 \\
\hline $\mathrm{B}$ & 8 & 10,3 \\
\hline C & 1 & 1,3 \\
\hline $\mathrm{D}$ & 4 & 5,1 \\
\hline$E$ & 2 & 2,6 \\
\hline $\mathrm{F}$ & 3 & 3,8 \\
\hline Componentes da aptidão física & Média & DP \\
\hline Flexibilidade $(\mathrm{cm})$ & 39,48 & 12,84 \\
\hline Coordenação (s) & 48,45 & 28,78 \\
\hline Agilidade (s) & 79,44 & 64,23 \\
\hline Força (repetições) & 8,28 & 5,02 \\
\hline Resistência aeróbia - TC6M (m) & 263,82 & 117,08 \\
\hline IAFG & 269,21 & 92,52 \\
\hline
\end{tabular}

$\mathrm{cm}$ : centímetros; DP: desvio-padrão; m: metros; s: segundos; TC6M: teste de caminhada de 6 minutos.

* Capacidade funcional: A - independente para todas as atividades; B - independente para todas as atividades menos uma; C - independente para todas as atividades menos banho e mais uma adicional; D - independente para todas as atividades menos banho, vestir-se e mais uma adicional; E - independente para todas as atividades menos banho, vestirse, ir ao banheiro e mais uma adicional; F - independente para todas as atividades menos banho, vestir-se, ir ao banheiro, transferência e mais uma adicional.

força $(r h o=-0,387 ; \mathrm{p}=0,001)$ e para o IAFG $(r h o=$ $-0,381 ; p=0,001)$; e positiva para a coordenação $(r h o=0,416 ; \mathrm{p}=0,001)$ e para a agilidade $(r h o=$ $0,372 ; \mathrm{p}=0,001)$. Assim, quanto maior o grau de dependência dos idosos institucionalizados menor é a força e o resultado do IAFG e, quanto melhor é a coordenação e a agilidade melhor é o nível de independência para a realização das atividades da vida diária.

\section{Discussão}

Ao explorar a relação entre aptidão física e a capacidade funcional de residentes em instituições de longa permanência para idosos de baixa ren- da, verificou-se que houve correlação moderada negativa para a força e o IAFG e, positiva para a coordenação e agilidade.

Portanto, quanto maior o grau de dependência dos idosos institucionalizados menor é a força e o resultado do IAFG e, quanto melhor é a coordenação e a agilidade/equilíbrio dinâmico melhor é o nível de independência para a realização das atividades da vida diária.

Desse modo, observa-se que uma boa aptidão física irá desencadear uma boa capacidade funcional, o que se confirmou neste estudo, por meio de uma correlação moderada, principalmente nos componentes da aptidão física, como a força, coordenação e agilidade/equilíbrio dinâmico, e no IAFG. 
Correlação entre os componentes da aptidão física, o Índice de Aptidão Funcional Geral (IAFG) e a capacidade funcional dos idosos residentes em instituições de longa permanência, 2009.

\begin{tabular}{lcc}
\hline Variáveis & rho Capacidade funcional & Valor de $p$ \\
& & \\
\hline Componentes da aptidão física & $-0,008$ & 0,948 \\
Flexibilidade (cm) & 0,416 & $<0,001$ \\
Coordenação (s) & 0,372 & $<0,001$ \\
Agilidade (s) & $-0,387$ & $<0,001$ \\
Força (repetições) & $-0,078$ & 0,497 \\
Resistência aeróbia - TC6M (m) & $-0,381$ & $<0,001$ \\
IAFG & & \\
\hline
\end{tabular}

cm: centímetros; m: metros; rho: correlação de Spearman s: segundos; TC6M: teste de caminhada de 6 minutos.

A aptidão física e seus componentes estão relacionados à saúde, pois bons índices estão associados com um menor risco de desenvolvimento de doenças e/ou incapacidades funcionais 15,16 . Essa aptidão é constantemente recrutada na realização de atividades do cotidiano, como levantar-se da cadeira, desviar de obstáculos e pessoas, andar pela casa, subir degraus, atravessar a rua em uma velocidade segura, entre outras 5,17. O declínio das aptidões como força, agilidade, flexibilidade e coordenação, faz com que o idoso tenha dificuldade em combinar movimentos, o que lhe exige muito esforço para realizar estas atividades do cotidiano 18 .

A resistência de força dos membros superiores é um fator importante para o IAFG, pois a fraqueza muscular pode avançar até que a pessoa não consiga realizar as atividades cotidianas, como levantar-se de uma cadeira ou transpor objetos 18 . A musculatura, quando recrutada com maior freqüência, torna-se mais firme, forte e flexível em comparação com os músculos inativos, que ficam fracos, flácidos e menos elásticos 19.

Para realizar movimentos corporais rápidos e de curta duração, com mudanças de direção e alterações na altura do centro de gravidade, nos quais, normalmente ocorrem acelerações e desacelerações, os idosos devem possuir bons níveis de agilidade/equilíbrio dinâmico. Esses níveis podem auxiliar no deslocamento em calçadas com desníveis e buracos e para desviar de pessoas e de objetos 15,19. Bons níveis de coordenação também contribuem nas atividades da vida diária como alimentar-se, abrir portas, manusear objetos etc.

No presente estudo, a flexibilidade não se correlacionou com a capacidade funcional dos idosos que residem em instituições, e ela é considerada de suma importância para os idosos, pois sua diminuição acarreta dificuldades para calçar sapatos, vestir-se, além de aumentar o risco de lesões e quedas, principalmente porque a flexibilidade dos membros inferiores está relacionada às mudanças no padrão de marcha 15,19,20.

Outra aptidão física que não apresentou correlação com a capacidade funcional no nosso estudo foi a resistência aeróbia, com base no TC6M. A taxa de declínio da resistência aeróbia nos idosos geralmente chega até $1 \%$ ao ano (em $\mathrm{VO}_{2 \text { máx }}$ ) e é maior em idosos sedentários 20.

No nosso estudo a média da resistência aeróbia, por meio do TC6M, foi de 263,82m. Esse resultado é inferior quando comparado à média da distância percorrida por idosos de diferentes estudos: $499 \mathrm{~m}$ realizados por 23 idosos com média de 65 anos de idade ( $\mathrm{DP}=7,6$ ), portadores de doença pulmonar obstrutiva crônica 21 ; média de $501,1 \mathrm{~m}$ percorridos por 22 idosas voluntárias da comunidade, independentes paraas atividades da vida diária e atividades instrumentais do cotidiano, praticantes de atividades físicas de lazer três vezes na semana por pelo menos 30 minutos 22; média de $486 \mathrm{~m}$ realizados por idosos institucionalizados canadenses, com idades entre 6089 anos 23; média de $603 \mathrm{~m}$ percorridos por 156 idosos institucionalizados da Bélgica, funcionalmente independentes 24 .

Em relação aos outros componentes da aptidão física dos idosos institucionalizados, avaliados por meio da bateria de testes para idosos, desenvolvida pela AAHPERD, ainda são escassos os estudos. Desse modo, as discussões dos resultados do nosso estudo terão como base a comparação com os valores de amplitude propos- 
tos originalmente pela bateria da AAHPERD 13 e com outros estudos que desenvolveram valores normativos para idosos ativos brasileiros 18,25 , conforme a Tabela 4.

Observa-se que os idosos do nosso estudo apresentam flexibilidade e força dentro do esperado por Osness et al. 13 e, coordenação e agilidade e equilíbrio dinâmico com amplitudes maiores que a prevista no artigo original, com limites inferiores e superiores maiores, ou seja, os resultados são piores. No entanto, os resultados da AAHPERD não provêm de idosos institucionalizados.

Também os valores da flexibilidade para os idosos de instituições de longa permanência foram inferiores aos apresentados para idosas brasileiras com idades entre 60-69 anos 18 e entre 707925 , porém, o limite inferior da amplitude ficou superior ao das idosas entre 60-69 anos.

A coordenação, agilidade e equilíbrio dinâmico e a força de membros superiores dos idosos do presente trabalho apresentaram piores resultados do que as idosas de outros estudos 18,25. Os estudos apresentados são com idosas independentes e fisicamente ativas, assim, é esperada essa diferença de valores, pois a amostra do presente trabalho é institucionalizada e sedentária.

Outro aspecto a ser considerado é a classificação dos componentes da aptidão física dos idosos institucionalizados e do IAFG. A flexibilidade, coordenação, agilidade, resistência aeróbia e o IAFG foram considerados regulares e a força boa.

Comparando os resultados dos idosos institucionalizados do nosso estudo com idosos ativos fisicamente, observa-se que os componentes da aptidão física, como a flexibilidade 26,27 , a agilidade e equilíbrio dinâmico, a força, a coordenação, a resistência aeróbia e o IAFG foram classificados como bom 26. Demonstrando com isso que os idosos das instituições de longa permanência devem realizar exercícios físicos para melhorar as suas aptidões físicas, visto que apenas um idoso dentre os 78 pesquisados praticava atividade física. Ao estudar idosas institucionalizadas verificou que a maioria é pouco ativa fisicamente 28 . As instituições de longa permanência para idosos não realizam atividades ocupacionais durante $o$ dia-a-dia dos idosos, fazendo com que se restrinjam às atividades menos exigentes e que requeiram menor esforço ${ }^{29}$. Também as atividades de lazer são limitadas e/ou ausentes nas instituições de longa permanência para idosos, o que favorece o sedentarismo que pode ser agravado com o aumento da idade, ocasionando a perda da aptidão física e o comprometimento da capacidade funcional 6 .

Diante do sedentarismo dos idosos das instituições de longa permanência, é necessário criar estratégias para incentivar a prática de exercícios físicos, visto os seus benefícios para a manutenção e melhoria da aptidão física e da capacidade funcional 30,31.

\section{Considerações finais}

Entre a capacidade funcional, a aptidão física em seus componentes e o IAFG foi encontrada uma relação moderada. Portanto, quanto maior o grau de dependência dos idosos institucionalizados menor é seu desempenho na capacidade força, repercutindo em índice geral mais baixo de aptidão física, enquanto melhor é o desempenho na coordenação e agilidade maior é o nível de independência funcional para a realização das atividades da vida diária.

Guardadas as limitações do presente estudo de impossibilidade de generalização de seus resultados, pode-se, contudo, inferir para fins práticos que é possível avaliar os componentes da aptidão física entre idosos institucionalizados, funcionalmente independentes ou em depen-

Tabela 4

Amplitudes de resultados propostos por diferentes autores e no presente estudo, para os testes da bateria da AAHPERD (American Alliance for Health, Physical Education, Recreation and Dance).

\begin{tabular}{lcccc}
\hline Estudos & Flexibilidade $(\mathbf{c m})$ & Coordenação (s) & Agilidade (s) & Força (repetições) \\
\hline Osness et al. 13 & $12,7-76,2$ & $8-25$ & $15-35$ & $0-40$ \\
Zago \& Gobbi 19 & $11,5-82,5$ & $7,7-25,3$ & $10,3-44,4$ & $10-43$ \\
Benedetti et al. 26 & $35,5-85,0$ & $7,5-27,5$ & $15,3-51,4$ & $10-37$ \\
Presente estudo & $15-65$ & $10-139$ & $20,4-519,3$ & $0-32$ \\
\hline
\end{tabular}

$\mathrm{cm}$ : centímetros; s: segundos. 
dência parcial, a fim de avaliar os déficits específicos passíveis de intervenção preventiva ou reabilitadora. É imprescindível a implementação de programa apropriado de atividades e exercícios físicos em instituições de longa permanência para idosos em busca da manutenção e/ou recuperação do processo de envelhecimento mais ativo, considerando a necessidade e importância da avaliação acurada por meio de instrumentos como a AAHPERD que discrimina o déficit ou não nos diferentes componentes da aptidão física, avançando para além dos resultados que se obtém em instrumentos mais gerais que avaliam a capacidade funcional em termos de dependência/independência para as atividades da vida diária em três níveis: dependente, parcialmente dependente e independente.

\section{Resumo}

O objetivo deste estudo foi explorar a relação entre a aptidão física e a capacidade funcional de residentes em instituições de longa permanência para idosos de baixa renda. Foi realizada avaliação em seis instituições localizadas em três regiões do país. Amostra foi composta de 78 idosos, com média de idade de 77,4 anos (DP = 7,9). A avaliação da aptidão física aplicando-se testes da AAHPERD adaptada para idosos institucionalizados, e da capacidade funcional pela escala de Katz, constatou que a aptidão física, em seus cinco componentes, em média era regular na flexibilidade, coordenação, agilidade e resistência aeróbia, era boa no componente força. Já o Índice de Aptidão Física
Geral (IAFG), em média era regular. Os resultados demonstram que quanto maior o grau de dependência dos idosos institucionalizados menor é a força e o resultado do IAFG e, quanto melhor é a coordenação e a agilidade melhor é o nível de independência para a realização das atividades da vida diária. As implicações estão em contribuir com os programas de exercícios físicos adequados na manutenção elou recuperação da funcionalidade.

Aptidão Física; Instituições de Longa Permanência para Idosos; Idoso 


\section{Colaboradores}

G. Z. Mazo, L. H. T. Gonçalves, A. H. Silva, T. R. B. Benedetti, R. A. P. Rodrigues, e M. Creutzberg participaram da concepção e planejamento, análise e interpretação dos dados, elaboração e revisão crítica do conteúdo e organização final do manuscrito. S. M. A. Santos, S. Marques, M. R. Portella, H. M. Scortegagna, S. S. C. Santos, M. T. Pelzer, A. S. Souza, E. C. Meira, E. L. S. Sena, e T. L. Rezende colaboraram na análise e interpretação dos dados, elaboração da revisão crítica do conteúdo e organização final do manuscrito.

\section{Referências}

1. Instituto Brasileiro de Geografia e Estatística. Síntese de indicadores sociais, 2006. Rio de Janeiro: Instituto Brasileiro de Geografia e Estatística; 2006.

2. Yamamoto A, Diogo MJD. Os idosos e as instituições asilares do Município de Campinas. Rev Latinoam Enferm 2002; 10:660-6.

3. Gierveld, Valk H, Blommesteijn M. Living arrangements of older persons and family support in more developed countries. New York: United Nations; 2001. (Population Bulletin of the United Nations, 42/43).

4. Caldas CP. Envelhecimento com dependência: responsabilidades e demandas da família. Cad Saúde Pública 2003; 19:733-81.

5. Christensen U, Stovring N, Schultz-Larsen K, Schroll M, Avlund K. Functional ability at age 75: is there an impact of physical inactivity from middle age to early old age? Scand J Med Science Sports 2006; 16:245-51.

6. Benedetti TRB, Mazo GZ, Schmitz LTM. Instituições geriátricas da grande Florianópolis. Arq Geriatr Gerontol 2000; 4:57-61.

7. Born T, Boechat NS. A qualidade dos cuidados ao idoso institucionalizado. In: Freitas EV, Neri AL, organizadores. Tratado de geriatria e gerontologia. Rio de Janeiro: Editora Guanabara Koogan; 2002. p. 1131-41.

8. Gomes GC, Diogo MJD. Função motora, capacidade funcional e sua avaliação em idosos. In: Diogo MJD, Neri A, Cachioni M, organizadores. Saúde e qualidade de vida na velhice. São Paulo: Alínea; 2004. p. 107-32.

\section{Agradecimentos}

Ao Conselho Nacional de Desenvolvimento Científico e Tecnológico (CNPq) e ao Ministério da Saúde pelo financiamento da pesquisa, processo 555079/2006-6. Aos bolsistas integrantes do processo 555079/2006-6, que atuaram nos seis centros, coletando dados e desempenhando funções próprias de bolsistas de iniciação científica: Murilo Alves, Rosely Souza, Ayrã Neves Assunção, Thaise Souza Oliveira, Talita Maiara, Russilene Lopes, Clariana V. Ramos, Monica Blanco, Cinara Borges Souza, Jamille Munhos Brahim, Ana Eugenia A. Bastos, Cleber Mossini; e aos bolsistas de apoio técnico: Moema Santos Souza, Daniela Silva Reis, Barbara Tarouco, Rochele Gampert Cruz, Marcia Welfer, Alice Carvalho Borsatto, Luiza Valmorbida, Thais Ramos, Anna Paula Xavier.

9. Freire Júnior RC, Tavares MFL. A promoção da saúde nas instituições de longa permanência: uma reflexão sobre o processo de envelhecimento no Brasil. Rev Bras Geriatr Gerontol 2006; 9:83-92.

10. Agência Nacional de Vigilância Sanitária. Resolução $n^{\circ} .283 / 05$. Dispõe sobre as instituições de longa permanência para idosos. Diário Oficial da União 2005; 26 set.

11. Lino VTS, Pereira SRM, Camacho LAB, Ribeiro Filho ST, Buksman S. Adaptação transcultural da Escala de Independência em Atividades da Vida Diária (Escala de Katz). Cad Saúde Pública 2008; 24:103-12.

12. Departamento de Atenção Básica, Secretaria de Atenção à Saúde, Ministério da Saúde. Envelhecimento e saúde da pessoa idosa. Brasília: Ministério da Saúde; 2006. (Série A. Normas e Manuais Técnicos) (Cadernos de Atenção Básica, 19).

13. Osness WH, Adrian M, Clark B, Hoeger W, Raab D, Wiswell R. Functional fitness assessment for adults over 60 years (a field based assessment). Reston: American Alliance for Health, Physical Education, Recreation and Dance; 1990.

14. ATS Committee on Proficiency Standards for Clinical Pulmonary Function Laboratories. ATS statement: guidelines for the six-minute walk test. Am J Respir Crit Care Med 2002; 166:111-7.

15. American College of Sports Medicine; ChodzkoZajko WJ, Proctor DN, Fiatarone Singh MA, Minson CT, Nigg CR, et al. American College of Sports Medicine position stand: exercise and physical activity for older adults. Med Sci Sports Exerc 2009; 41:1510-30. 
16. Glaner MF. Importância da aptidão física relacionada à saúde. Rev Bras Cineantropom Desempenho Hum 2003; 5:75-85.

17. Silva CM, Gurjão ALD, Ferreira L, Gobbi LTB, Gobbi S. Efeito do treinamento com pesos, prescrito por zonas de repetições máximas, na fora muscular e composição corporal em idosas. Rev Bras Cineantropom Desempenho Hum 2006; 8:39-45.

18. Zago AS, Gobbi S. Valores normativos da aptidão funcional de mulheres de 60 a 70 anos. Rev Bras Ciênc Mov 2003; 11:77-86.

19. Gobbi S, Villar R, Zago AS. Bases teórico-práticas do condicionamento físico. Rio de Janeiro: Editora Guanabara Koogan; 2005.

20. Spirduso WW. Physical dimensios of aging. Champaing: Human Kinetics; 1995.

21. Moreira MAC, Moraes MR, Tannus R. Teste da caminhada de seis minutos em pacientes com DPOC durante programa de reabilitação. J Bras Pneumol 2001; 27:295-300.

22. Geraldes AAR, Albuquerque RB, Soares RM, Carvalho J, Farinatti PTV. Correlação entre flexibilidade das articulações glenoumerais e coxofemorais e o desempenho funcional de idosas fisicamente ativas. Rev Bras Fisioter 2008; 12:274-82.

23. Steffen TM, Hacker TA, Mollinger L. Age- and gender-related test performance in communitydwelling elderly people: six-minute walk test, berg balance scale, timed up \& go. Phys Ther 2002; 82:128-37.

24. Bautmans I, Lambert M, Mets T. The six-minute walk test in community dwelling elderly: influence of health status. BMC Geriatr 2004; 4:6.
25. Benedetti TRB, Mazo GZ, Gobbi S, Lopes MA, Gobbi LTB, Ferreira L, et al. Valores normativos de aptidão funcional em mulheres de 70 a 79 anos. Rev Bras Cineantropom Desempenho Hum 2007; 9:28-37.

26. Sebastião E, Hamanaka AYY, Gobbi LTB, Gobbi S. Efeitos da prática regular de dança na capacidade funcional de mulheres acima de 50 anos. Rev Educ Fís 2008; 19:205-14.

27. Rosa TEC, Benício MHD, Latorre MRDO, Ramos LR. Fatores determinantes da capacidade funcional entre idosos. Rev Saúde Pública 2003; 37:40-8.

28. Benedetti TRB, Petroski EL, Gonçalves LT. Exercí cio físico e atividades da vida diária em idosos asilados. Texto \& Contexto Enferm 2001; 10:52-67.

29. Benedetti TRB, Petroski EL. Idosos asilados e a prática da atividade física. Rev Bras Ativ Fís Saúde 1999; 4:5-16.

30. Rolland Y, Pillard F, Klapouszczak A, Reynish E, Thomas D, Andrieu S, et al. Exercise program for nursing home residents with Alzheimer's disease: a 1-year randomized, controlled trial. J Am Geriatr Soc 2007; 55:158-65.

31. Vreede PL, Samson MM, Meeteren NLU, Duursma SA, Verhaar HJJ. Functional-task exercise versus resistance strength exercise to improve daily function in older women: a randomized, controlled trial. J Am Geriatr Soc 2005; 53:2-10.

Recebido em 23/Dez/2009

Versão final reapresentada em 26/Jul/2010

Aprovado em 02/Ago/2010 